\title{
A. SPHERICAL MAPPING AND BORSUK CONJECTURE IN RIEMANNIAN AND NON-EUCLIDEAN SPACES
}

\author{
BORIS V. DEKSTER
}

\begin{abstract}
We introduce an analog of the spherical mapping for convex bodies in a Riemannian $n$-manifold, and then use this construction to prove the Borsuk conjecture for some types of such bodies. The Borsuk conjecture is that each bounded set $X$ in the Euclidean $n$-space can be covered by $n+1$ sets of smaller diameter. The conjecture was disproved recently by Kahn and Kalai. However Hadwiger proved the Borsuk conjecture under the additional assumption that the set $X$ is a smooth convex body. Here we extend this result to convex bodies in Riemannian manifolds under some further restrictions.
\end{abstract}

We introduce here an analog of spherical mapping for convex bodies in a Riemannian $n$-manifold $M^{n}$ and then use this construction to prove the Borsuk conjecture for some types of such bodies. The Borsuk conjecture stated by him in 1933 is that each bounded set $X$ in $R^{n}$ can be covered by $n+1$ sets of smaller diameter. The conjecture was disproved recently by Kahn and Kalai [9]. For more on the history of the problem and its partial solutions, see [9] and the references there. In particular, Hadwiger $[6,7,8]$ proved the Borsuk conjecture under the additional assumption that the set $X$ is a smooth convex body. Here, in Theorem 1, we extend this result to convex bodies in Riemannian manifolds under some further restrictions.

This paper is closely related to [4]. We remind now the basic definitions from there.

The manifold $M^{n}$ is supposed to be regular but not necessarily complete. A set $C \subset M^{n}$ will be called definitely convex if

(i) $C$ is compact and each two points of $C$ can be connected in $C$ by a rectifiable curve;

(ii) Any shortest path in $C$ between two points of $C$ is a geodesic. (The path exists due to (i).);

(iii) Any geodesic segment in $C$ is the unique shortest path in $C$ between its ends;

(iv) Each geodesic segment in $C$ contains no pair of conjugate points along this segment.

Received December 14, 1992; revised March 13, 1993.

1991 Mathematics Subject Classification. Primary 52A20, 52A37, 52A55, 53C99.

Key words and phrases. Convex bodies in manifolds, spherical mapping, Borsuk conjecture.

Supported by a Canadian NSERC Grant. 
Some examples of definitely convex sets can be found in the beginning of [4]. Let $C$ be a definitely convex set. If $C$ is homeomorphic to a ball, it will be called a definitely convex body. It can be shown [4, the beginning] that if the set $C$ has an interior point, then it is a definitely convex body.

We denote by $x y$ both a closed geodesic segment with the ends $x, y$ and its length. The meaning will be specified in case of possible confusion. Throughout the paper, we will deal with exterior unit normals of convex bodies. For short, we call them just normals.

Let $C \subset M^{n}$ be a definitely convex body, $a, b \in \partial C$ and $n_{a}, n_{b}$ be normals at $a$ and $b$ respectively (defined for instance in $[2, \$ 4.5]$ ). Suppose that the parallel translation along the chord $a b$ turns $n_{a}$ into $-n_{b}$. Then the normals $n_{a}$ and $n_{b}$ are said to be antipodal and the points $a, b$ antipodes. (Symmetry of this relation is obvious.)

Each normal $n_{c}$ has at least one antipodal normal ([4, Theorem 1] but can have more. Condition (iv) above is used in proof of this Theorem 1 in [4].

We construct now our spherical mapping for the definitely convex body $C \subset M^{n}$. Take a point $z \in C$ and denote by $S=S^{n-1}$ the unit sphere of directions at $z$. Let $N \subset T M^{n}$ be the set of all normals of $C$. For $v \in N$, denote by $A(v) \subset N$ the set of all normals antipodal to $v$. The notation $n_{x} \in N$ will imply that $n_{x}$ is a normal at a point $x \in \partial C$ or $\pi\left(n_{x}\right)=x$ where $\pi: T M^{n} \rightarrow M^{n}$ is the natural projection. Fix some $n_{x} \in N$ and pick up an $n_{y} \in A\left(n_{x}\right)$. Denote by $o$ the midpoint of the chord $x y$. Now let $\varphi\left(n_{x}, n_{y}\right) \in S$ be the result of parallel translation of the vector $n_{x}$ to the point $z$ along the polygonal line $x o z$, see Fig. 1. (The shortest geodesic segments xo and oz exist and are unique due to (ii) and (iii).) Note that

$$
\varphi\left(n_{x}, n_{y}\right)=-\varphi\left(n_{y}, n_{x}\right)
$$

i.e., $\varphi\left(n_{x}, n_{y}\right)$ and $\varphi\left(n_{y}, n_{x}\right)$ are antipodes on the sphere $S$.

Put

$$
\Phi\left(n_{x}\right)=\cup_{n_{y} \in A\left(n_{x}\right)} \varphi\left(n_{x}, n_{y}\right)
$$

thus defining a mapping

$$
\Phi: N \rightarrow 2^{S} \text {. }
$$

In Euclidean case, the image $\Phi(v), v \in N$, is always a single point of $S$ even when $v$ has more than one antipodal normals. We finally define the spherical mapping $\Omega: \partial C \rightarrow 2^{S}$ for $C$ with respect to $z$ putting

$$
\Omega(x)=\cup_{\pi(v)=x} \Phi(v), x \in \partial C .
$$

Remark 1. Let $x, y \in \partial C$ be antipodes. Then there exists a pair $n_{x}, n_{y}$ of antipodal normals. By (4) and (2),

$$
\Omega(x) \supset \Phi\left(n_{x}\right) \ni \varphi\left(n_{x}, n_{y}\right) ; \quad \Omega(y) \supset \Phi\left(n_{y}\right) \ni \varphi\left(n_{y}, n_{x}\right) .
$$




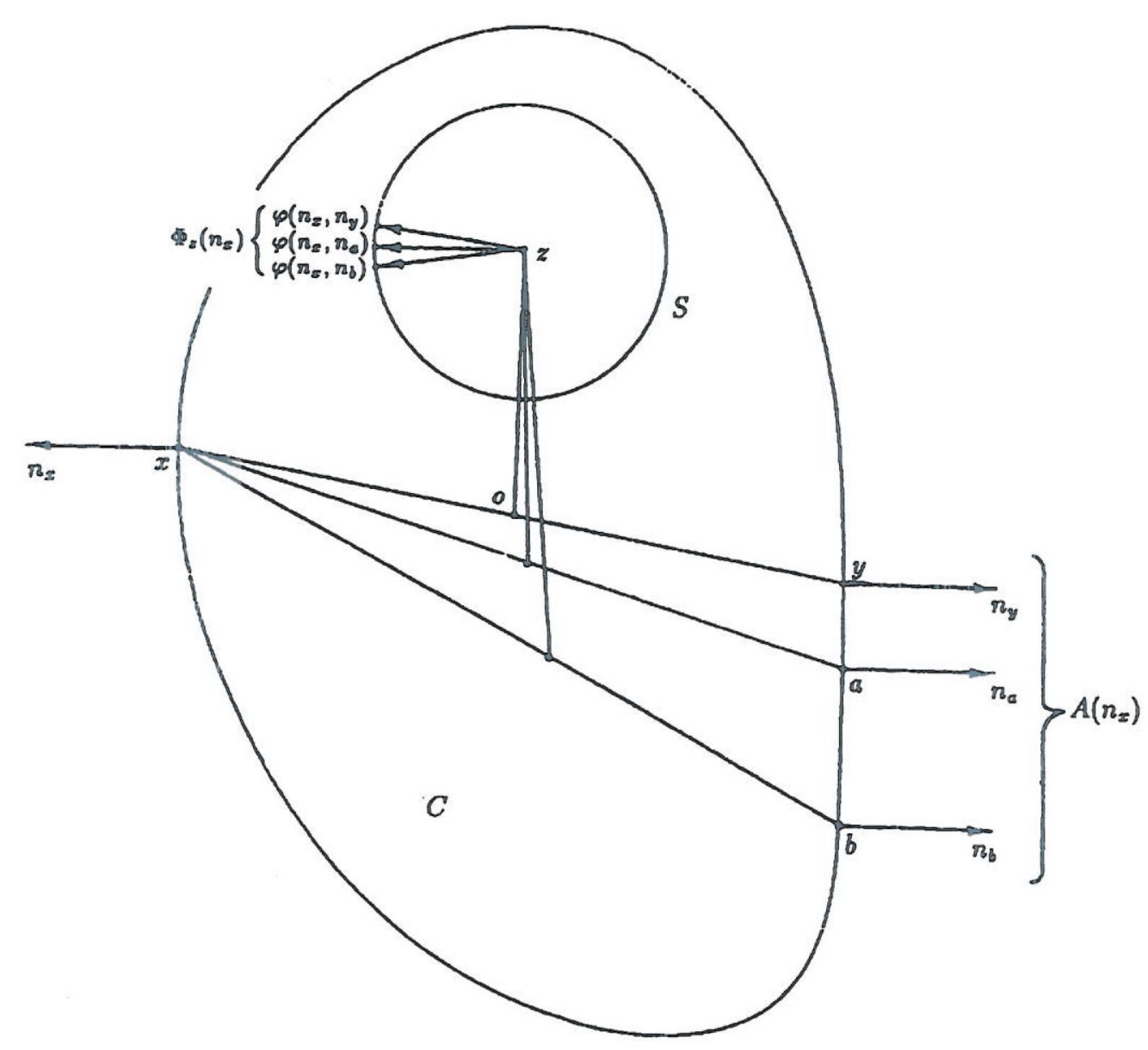

Fig. 1. The $A\left(n_{x}\right)$ and $\Phi_{z}\left(n_{x}\right)$. (here, each consists of three elements.)

Thus the sphere $S$ contains a pair of antipodes, $\varphi\left(n_{x}, n_{y}\right)$ and $\varphi\left(n_{y}, n_{x}\right)$, see (1), one in $\Omega(x)$, the other in $\Omega(y)$.

Remark 2. For a set $\Delta \subset S$, put

$$
\Omega^{-1}(\Delta)=\{x \in \partial C \mid \Omega(x) \bigcap \Delta \neq \phi\}
$$

We would like to show that $\Omega^{-1}(\Delta)$ is closed if $\Delta$ is closed. Suppose the contrary. Then $\partial C$ contains a sequence $x_{i}$ such that

$$
x_{i} \rightarrow_{i \rightarrow \infty} x \in \partial C, \quad \Omega\left(x_{i}\right) \bigcap \Delta \neq \phi, \quad \Omega(x) \bigcap \Delta=\phi .
$$

Let $\alpha_{i} \in \Omega\left(x_{i}\right) \cap \triangle$. By (4), $\alpha_{i}$ lies in a set $\Phi\left(n_{x_{i}}\right)$ and, by (2)

$$
\alpha_{i}=\varphi\left(n_{x_{i}}, n_{y_{i}}\right)
$$

for some normal $n_{y_{i}}$ (at a point $y_{i} \in \partial C$ ) antipodal to $n_{x_{i}}$ 
By compactness of $\partial C$ and $N$, one may assume that

$$
y_{i} \rightarrow y \in \partial C, n_{x_{i}} \rightarrow n_{x} \in N, n_{y_{i}} \rightarrow n_{y} \in N \quad \text { as } \quad i \rightarrow \infty
$$

where the limits $n_{x}$ and $n_{y}$ must be normals at $x$ and $y$ according to [4, Lemma 2].

Note that the shortest paths $x_{i} y_{i}$ converge to the path $x y$ due to (ii) and (iii). Condition (iv) and regularity (of a properly selected mapping) imply now easily that $n_{x}$ and $n_{y}$ are antipodal. By continuity,

$$
\alpha_{i} \rightarrow_{i \rightarrow \infty} \alpha \stackrel{\text { def }}{=} \varphi\left(n_{x}, n_{y}\right) \in \Phi\left(n_{x}\right) \subset \Omega(x) .
$$

Since $\triangle$ is closed and $\alpha_{i} \in \Delta$, one has $\alpha \in \Delta$. Along with (10), this means that $\alpha \in \Omega(x) \cap \triangle$ contrary to $(7)$.

Remark 3. The mapping $\Omega$ appears to be only a poor surrogate of the spherical mapping in $R^{n}$. It depends on the point $z$ and on the metric deep inside $C$. Moreover, even when each normal has a unique antipodal one, the normal cone at a boundary point 2 generally speaking is not isometric to the cone in $T_{z} M^{n}$ determined by $\Omega(x)$. However the properties of $\Omega$ established in Remarks 1 and 2 are sufficient for our objectives.

Theorem 1. Let $C \subset M^{n}$ be a definitely convex body whose normal is unique at every boundary point. (I.e., each point of $\partial C$ is regular or $C$ is smooth.) Suppose that, for each segment $a b \subset C$ of length diam $C$, the direction of $a b$ at $b$ is a unique antipodal normal of the direction of ba at a. (As in [3, Remark 3], one can check that $a, b \in \partial C$ and both directions above are normals of $C$.) Then there exist compact sets $Q_{i} \subset C, i=1,2, \cdots, n+1$, such that $C=\cup_{i=1}^{n+1} Q_{i}$ and diam $Q_{i}<$ diam $C$ for each $i$. (Thus the Borsuk conjecture holds for $C$.)

Remark 4. Note that for sufficiently big sets in $M^{n}$, the Borsuk conjecture can easily fail. Consider for instance the set of 4 vertices of the standard partition of $S^{2}$ into 4 equal triangles.

Remark 5. The uniqueness of an antipodal normal assumed in Theorem 1 can easily fail even in a simple case of $M^{n}=H^{2}$ (hyperbolic plane). Consider in $H^{2}$ a triangle $a b c$ with $\angle b a c=\angle a c b=40^{\circ}$ and $a b=b c>a c$. Its side $a b$ is one of its diameters. The direction $n_{a}$ of the side $b a$ at $a$ has two antipodal normals: the direction $n_{b}$ of $a b$ at $b$ and the vector $n_{c}$ at $c$ which forms angles $100^{\circ}$ and $140^{\circ}$ with the sides $c b$ and $c a$ respectively. The triangle in this example can be easily replaced by a smooth body.

It is not a simple task to check the uniqueness above directly. We do not know however any convenient sufficient conditons of this uniqueness which would not be too restrictive. Some rather restrictive conditions of this sort yield the following.

Theorem 2. Let $C$ be a smooth definitely convex body in a manifold $M_{k}^{n}$ of constant curvature $k \in\{-1,0,1\}$. Suppose that

$$
\cosh (\operatorname{diam} C) \leq 2 \text { if } k=-1 \text {. }
$$


then there exist compact sets $Q_{i} \subset C, i=1,2, \cdots, n+1$, such that $C=\cup_{i=1}^{n+1} Q_{i}$ and $\operatorname{diam} Q_{i}<\operatorname{diam} C$ for each $i$.

Proof of $\mathrm{Theorem} 1$. It follows the ideas of Hadwiger as presented in $[1, \S 6$, proof of Theorem 4]. Let $S=\cup_{i=1}^{n+1} \triangle_{i}$ be the standard partition of $S$ into $n+1$ equal spherical $(n-1)$-simplexes $\triangle_{i}$. Then

$$
\operatorname{diam} \triangle_{i}<\pi, \quad i=1,2, \cdots, n+1 .
$$

Put $P_{i}=\Omega^{-1}\left(\triangle_{i}\right)$. Let us show that

$$
\operatorname{diam} P_{i}<d \stackrel{d e f}{=} \operatorname{diam} C .
$$

(Both diameters are measured in $M^{n}$.) Suppose to the contrary that diam $P_{i}=d$. Since $P_{i}$ is compact (see Remark 2), there exists a chord $a b$ with $a, b \in P_{i}$ of the length $d$. The direction $n_{a}$ of the chord $b a$ at $a$ is the unique normal of $C$ at $a$ and the direction $n_{b}$ of the chord $a b$ at $b$ is the unique normal at $b$. Obviously $n_{a}$ and $n_{b}$ are antipodal. By our assumption, each of them has no other antipodal normals. Obviously $\Omega(a)$ is a point, $\Omega(b)$ is a point, and both points lie in $\triangle_{i}$. By Remark 1 , they are antipodes on the sphere $S$. This contradicts (12).

Clearly, $\partial C=\cup_{i=1}^{n+1} P_{i}$. Fix a point $q \in \operatorname{int} C$ and let $Q_{i}$ be the union of all the segments $p q$ with $p \in P_{i}$. We leave it to the reader to show that $C=\cup_{i=1}^{n+1} Q_{i}$. Obviously $Q_{i}$ is compact. Therefore there exists a segment $x y$ with $x, y \in Q_{i}$ of length $d_{i}=\operatorname{diam} Q_{i}$. Let $p q, p \in P_{i}$, be a segment which contains an end of the diameter $x y$. By [5, Lemmas 3 and 1], the set $p q \backslash p \subset$ int $C$. If our end of $x y$ differs from $p$ then the segment $x y$ can be extended within $C$ still remaining a shortest path according to (iii). In this case, $d_{i}<d$. The only remaining possibility is that both $x$ and $y \in P_{i}$. Then $d_{i}<d$ by (13). This completes the proof.

Proof of Theorem 2. The proof follows obviously from Theorem 1 and the following

Lemma. Let $C$ be a definitely convex body (not necessarily smooth) in an $n$-manifold $M_{k}^{n}$ of constant curvature $k \in\{-1,0,1\}$. Suppose that (11) holds: $\cosh (\operatorname{diam} C) \leq 2$ if $k=-1$. Then, for each segment ab $\subset C$ of length diam $C$, the direction of $a b$ at $b$ is a unique antipodal normal of the direction of ba at $a$.

Proof. Note first that though $M_{k}^{n}$ is not necessarily $H^{n}, R^{n}$, or $S^{n}$, the body $C$ can be isometrically embedded in there by means of an "exponential type of mapping". Therefore the appropriate trigonometry can be used for the triangles within $C$.

Denote by $n_{a}$ and $n_{b}$ the two normals mentioned in the Lemma. Suppose to the contrary that a normal $n_{c}$ at $c \neq b$ is antipodal to $n_{a}$. Denote by $t$ the length of the chord $a c$, by $\alpha$ the angle $\angle b a c$ and by $\gamma$ the angle $\angle a c b$ of the triangle $a b c$, see Figure 2 . Obviously 


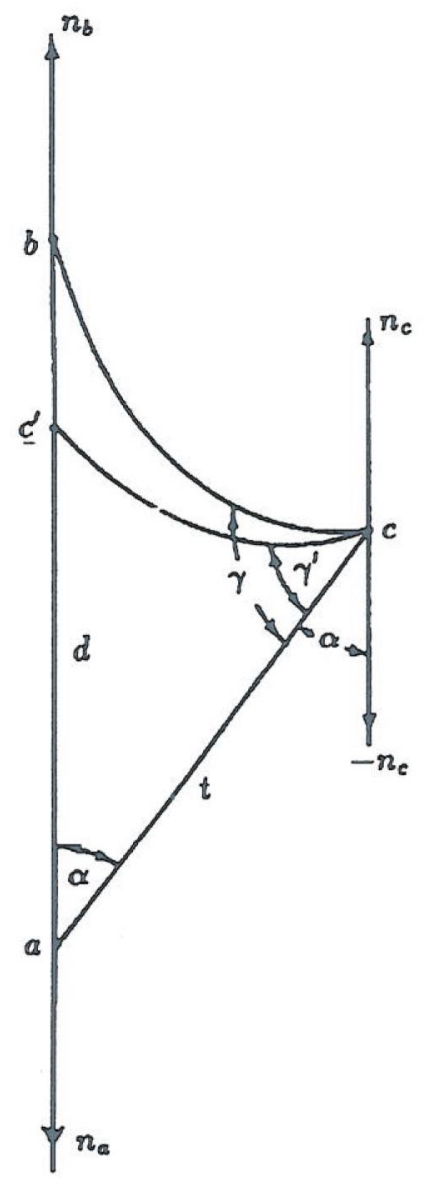

Fig. 2. The normals $n_{b}$ and $n_{c}$ are antipodal to $n_{a}$.

$$
0<\alpha<\pi / 2 ; \quad 0<t \leq d=\operatorname{diam} C .
$$

Denote by $M_{k}^{2}$ the plane of the triangle abc. Obviously the vector $n_{a}$ is tangent to $M_{k}^{2}$. The vector $-n_{c}$, being the result of the parallel translation of $n_{a}$ along the segment ac $\subset M_{k}^{2}$, is also tangent to $M_{k}^{2}$. Hence the directions $u$ and $v$ of the segments $c a$ and $c b$ at $c$ and the vector $-n_{c}$ lie in the same 2-dimensional direction at $c$. Therefore the angles between the three vectors satisfy

$$
\angle\left(-n_{c}, u\right)+\angle(u, v)=\angle\left(-n_{c}, v\right) .
$$

The last angle, being the angle between the interior normal $-n_{c}$ and the chord $c b$ of the definitely convex body $C$, is $\leq \pi / 2$. The first angle $\angle\left(-n_{c}, u\right)=\alpha$ since $n_{c}$ is antipodal to $n_{a}$. Thus

$$
\alpha+\gamma \leq \pi / 2 .
$$

Take a point $c^{\prime}$ on the diameter $a b$ such that the lengths $a c^{\prime}=a c=t$. In the case $k=-1$, out of the triangle $a c c^{\prime}$, one has

$$
\cosh t \cdot \tan (\alpha / 2)=\cot \gamma^{\prime} \text { where } \gamma^{\prime}=\angle a c c^{\prime} .
$$


Obviously $\gamma^{\prime} \leq \gamma$. Now by (15),

$$
\gamma^{\prime} \leq \pi / 2-\alpha
$$

Taking cotangent of both parts and combining the result with (16), one has

$$
\begin{gathered}
\cosh t \cdot \tan (\alpha / 2) \geq \tan \gamma \\
\cosh t \geq 1+\sec \alpha>2 \text { for } \alpha \in(0, \pi / 2) .
\end{gathered}
$$

by (14),

$$
\cosh d \geq \cosh t>2
$$

which contradicts (11).

For the case $k=1$, replace cosh by cos everywhere up to (19) inclusive.

Then (19) will be a contradiction. The case $k=0$ is trivial.

\section{References}

[1] V. Boltjansky and I. Gohberg, Results and problems in Combinatorial Geometry, Cambridge University Press, Cambridge-London-New York, 1985.

[2] Yu. D. Burago and V. A. Zalgaller, "Convex sets in Riemannian spaces of non-negative curvature." Russian Math. Surveys, 32 (1977) no. 3, 1-57.

[3] B. V. Dekster, "Bodies of constant width in Riemannian manifolds and spaces of constant curvature," In: Applied Geometry and Discrete Mathematics, Victor Klee Festschrift, DIMACS series, V. 4, (1991), edited by Peter Gritzman and Bernd Strumfels, copublished by AMS and ACM, 181-192.

[4] B. V. Dekster, "Width and breadth for convex bodies in Riemanian manifolds." Archiv der Mathematik, Vol. 58, (1992), 190-198.

[5] B. V. Dekster, "Double normals characterize bodies of constant width in Riemannian manifolds." In: Geometric analysis and nonlinear partial differential equations. Editor: I. Ya. Bakelman. Marcel Dekker, New York-Basel-Hong Kong, (1993), 187-201.

[6] H. Hadwiger, "Überdeckung eine Menge durch Mengen kleineren Durchmessers." Comment. Math. Helv., 18, (1945/46), 73-75.

[7] H. Hadwiger, Mitteilung betreffend meiner Note: "Überdeckung eine Menge durch Mengen kleineren Duchmessers," Comment. Math. Helv., 19, (1946/47), 72-73.

[8] H. Hadwiger, "Über die Zerstückung eines Eikorpers," Math. Z. 51, (1947), 161-165.

[9] J. Kahn, and G. Kalai, "A counterexample to Borsuk's conjecture," Bulletin of AMS, vol.29, no.1 (1993), 60-62.

Department of Mathematics, Mount Allison University, Sackville, N.B. EOA 3CO, Canada. 